\title{
Modification of Interleukin-15 Serum Levels in Workers Exposed to Chemotherapeutic Agents
}

\author{
Giovanna Spatari, ${ }^{1}$ Concettina Fenga, ${ }^{1}$ Paola Lucia Minciullo, ${ }^{2}$ \\ Giuseppe Di Pasquale, ${ }^{3}$ Anna Cacciola, ${ }^{1}$ Elvira Ventura-Spagnolo, ${ }^{4}$ \\ and Sebastiano Gangemi² \\ ${ }^{1}$ Department of Social and Environmental Medicine - Section of Occupational Medicine, University of Messina, 98125 Messina, Italy \\ ${ }^{2}$ Department of Human Pathology, School of Allergy and Clinical Immunology, University of Messina, 98125 Messina, Italy \\ ${ }^{3}$ Department of Internal Medicine, University of Messina, 98125 Messina, Italy \\ ${ }^{4}$ Department of Hygiene, Preventive Medicine and Public Health "R. De Blasi," University of Messina, 98125 Messina, Italy
}

Received 21 October 2004; accepted 1 November 2004

Cytostatic anticancer drugs are known as carcinogenic, mutagenic, and teratogenic risk factors for health care workers occupationally exposed. It has been demonstrated that the administration of interleukin-15 in rat models of colon carcinoma protects against chemotherapy-induced gastrointestinal toxicities. We found that occupational exposure to chemotherapeutic antiblastic agents in vivo modified circulating levels of interleukin-15 in 17 health care workers exposed to antineoplastic drugs in relation to their jobs and in as many healthy age- and sex-matched subjects. Health care workers displayed significantly higher circulating interleukin-15 levels compared to their age-matched controls. If this increase representing an anticancer response remains to be established, these findings strengthen the idea of a therapeutic use of interleukin-15 in the field of cancer.

Cytostatic anticancer drugs are known as carcinogenic, mutagenic, and teratogenic risk factors for health care workers who are occupationally exposed during preparation and management of such drugs, absorbing these substances mainly through inhalation or dermal contact. Risk assessment for occupational exposure to antiblastic chemotherapeutic drugs is carried out by means of environmental and biological monitoring [1]. To reduce these risks during the last twenty years, several researchers have developed and validated methods for monitoring occupational exposure to such agents. In Italy, for example, a working group, Prevention of Occupational Risks Due to Handling Antineoplastic Drugs in Health Care, was established by the Italian Institute of Prevention and Safety at Work (Istituto Superiore per la Prevenzione e Sicurezza sul Lavoro (ISPESL)) in February 1995 to make recommendations for environmental and biological monitoring of exposure and health surveillance, and develop guidelines for primary and secondary prevention [2].

Interleukin (IL)-15 is a T cell growing factor implicated in inflammatory response of the host. It plays a key role in development, differentiation, and function of

Correspondence and reprint requests to Sebastiano Gangemi, Department of Human Pathology, School of Allergy and Clinical Immunology, University of Messina, 98125 Messina, Italy; gspatari@unime.it
$\mathrm{B}$ and $\mathrm{T}$ lymphocytes, natural killer (NK) cells, NK-T cells, macrophages, and monocytes [3]. IL-15 has biologic properties in vitro similar to IL-2, consistent with their shared receptor signaling component [3]. It was demonstrated that the administration of IL-15 in rat models of colon carcinoma protects against chemotherapy-induced gastrointestinal toxicities (reviewed in [3]).

In light of these findings, we found that occupational exposure to chemotherapeutic antiblastic agents modifies in vivo circulating levels of IL-15.

We studied 17 health care workers cases (11 men and 5 women) (Group A) with a mean age \pm SD $42.29 \pm 6.08$ years, who had been exposed to antineoplastic drugs in relation to their jobs for $13.25 \pm 8.16$ years. The task of the health care workers under study was the preparation and administration of the antineoplastic drugs at the oncological and hematological centers of two city hospitals.

A questionnaire was used to obtain information on medical and occupational history, drug usage, and smoking and drinking habits.

Seventeen healthy age- and sex-matched subjects (Group B), with a negative history for exposure to antineoplastic drugs, were also enrolled as controls.

Exclusion criteria in both groups were the following: inflammatory, immunologic, oncologic, or other serious diseases for which the patients have taken drugs likely to interfere with the study.

All subjects gave their informed written consent to participate in the study. 


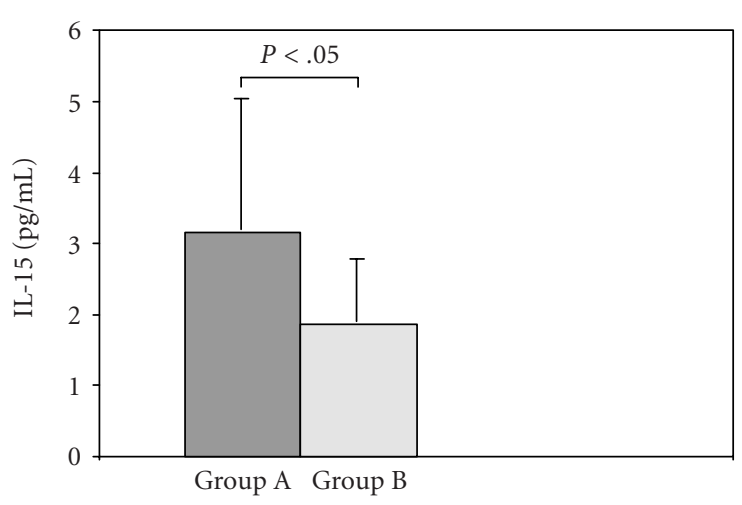

Figure 1. IL-15 serum levels in workers exposed to chemotherapeutic antiblastic agents (Group A), and in healthy donors (Group B). Results, expressed as mean \pm standard deviations, are from individual measurements in duplicate.

A $10 \mathrm{~mL}$ blood sample was collected from the antecubital vein, allowed to clot at room temperature for 2 hours, separate by centrifugation at $200 \mathrm{xg}$ for 15 minutes in a 4235 A centrifuge (ALC Int. SRL, Milan, Italy) and stored at $80^{\circ} \mathrm{C}$ until use.

Serum IL-15 levels were assayed by immunoenzymatic methods (Quantikine Human IL-15, ELISA-kits, R\&D System, SPACE Import-Export, Milan, Italy). The minimum detectable dose of IL-15 is less than $2 \mathrm{pg} / \mathrm{mL}$.

Differences in serum levels were assessed by one way analysis of variance (ANOVA) and Student-NewmanKeuls test. Data were expressed as mean \pm SD). A value of $P<.05$ was considered significant.

Figure 1 shows that group A subjects displayed significantly higher circulating IL-15 levels compared to their age-matched controls $(3.15 \pm 1.88$ versus $1.87 \pm$ $89.16 \mathrm{pg} / \mathrm{mL}, P<.05)$.

No significant correlation was found between IL-15 serum levels and age and duration of antineoplastic drugs exposure.

IL-15 is known to improve the in vivo antitumor activity of adoptively transferred $\mathrm{CD}^{+} \mathrm{T}$ cells; in fact it helps to maintain a memory $\mathrm{CD}^{+} \mathrm{T}$ cell population and can inhibit apoptosis [4]. Moreover, it was found that endogenous production of IL-15, but not IL-2, by a tumorbearing host enhanced the in vivo antitumor efficacy of adoptively transferred $\mathrm{CD} 8^{+} \mathrm{T}$ cells [4].

Two studies demonstrated that IL-15 is able to provide significant protection from intestinal toxicity of two different chemotherapeutic agents, irinotecan (CPT-11) (reviewed in [3]) and 5-fluorouracil (reviewed in [3]) with maintenance and potentiation of antitumor activity, and an increased therapeutic index of the drugs administered. In addition, it was demonstrated that IL-15 administration associated with cyclophosphamide (CY) injection in a mouse lung tumor model resulted in a significant prolongation of life [5]. For successful CY + IL-15 therapy, the presence of NK and T lymphocytes seems to be necessary [5].
In the present study we found significantly higher serum levels of IL-15 in subjects with occupational exposure, during preparation and administration, to chemotherapeutic antiblastic drugs, with respect to nonexposed subjects. To the best of our knowledge there are no reports about the involvement of immune system during or after antiblastic drugs exposure in humans.

It is known that occupational exposure to antineoplastic agents may cause carcinogenic, mutagenic, and teratogenic effects, therefore there is, today, a general consensus on the necessity of monitoring occupational exposure to such agents. Health care workers of our study had an exposure time covering also the years before the guidelines application. Thus, it might be hypothesized that the increase of IL-15 that we found may be the expression of an endogenous production by human cells as antitumor response.

It is known that immunotherapy with IL-2 has been used extensively for the treatment of patients with cancer, even if some qualities may preclude it from being the optimal T cell growth/activation factor for use in immunotherapy [4]. IL-15 and IL-2 share similar properties and utilize the same affinity receptor complex, although they can play different, and at times oppositional, roles in $\mathrm{T}$ cell biology. Moreover, in murine models comparing treatment with IL-15 and IL-2 for efficacy against experimental lung metastasis, IL-15 showed a higher therapeutic index and a lower toxicity than IL-2 (reviewed in [3]). Therefore, it may be speculated that in a near future the in vivo antitumor activity of IL-15 in mice may be translated in humans.

In conclusion, here we provide the first evidence of a strong increase in circulating IL-15 in subjects with occupational exposure to chemotherapeutic antiblastic drugs.

If this increase representing an anticancer response remains to be established, these findings strengthen the idea of a therapeutic use of IL-15 in the field of cancer.

\section{REFERENCES}

[1] Merler E, Villa L, Lucchini R. Pathological effects due to antineoplastic drugs in workers engaged in their production, preparation or administration. Med Lav. 1996;87(3):207-221.

[2] Alessio L, Apostoli P, Draicchio F, et al. Prevention of risks from occupational exposures to antineoplasticdrugs: consensus document. Int J Occup Environ Health. 1997;3(1):84.

[3] Fehniger TA, Caligiuri MA. Interleukin 15: biology and relevance to human disease. Blood. 2001;97(1):14-32.

[4] Klebanoff CA, Finkelstein SE, Surman DR, et al. IL15 enhances the in vivo antitumor activity of tumorreactive CD8+ T cells. Proc Natl Acad Sci U S A. 2004;101(7):1969-1974.

[5] Chapoval AI, Fuller JA, Kremlev SG, Kamdar SJ, Evans R. Combination chemotherapy and IL-15 ad- 
ministration induce permanent tumor regression in a mouse lung tumor model: NK and $\mathrm{T}$ cellmediated effects antagonized by B cells. J Immunol. 1998;161(12):6977-6984. 


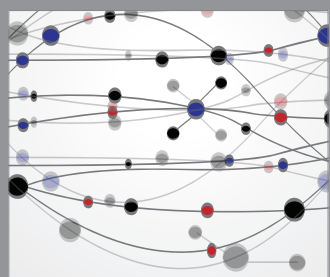

The Scientific World Journal
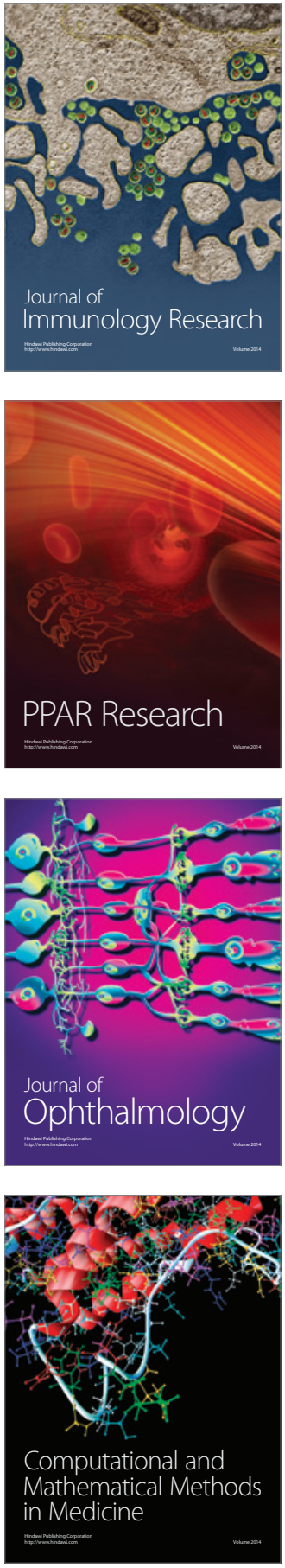

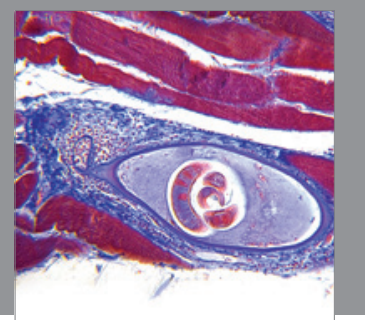

Gastroenterology

Research and Practice
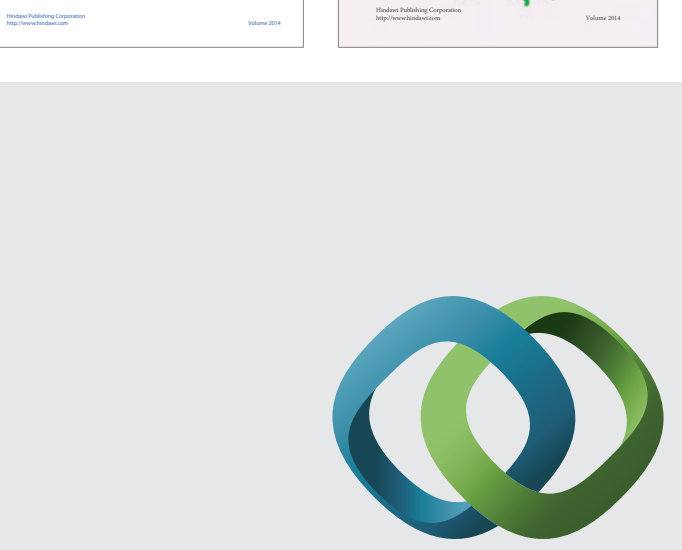

\section{Hindawi}

Submit your manuscripts at

http://www.hindawi.com
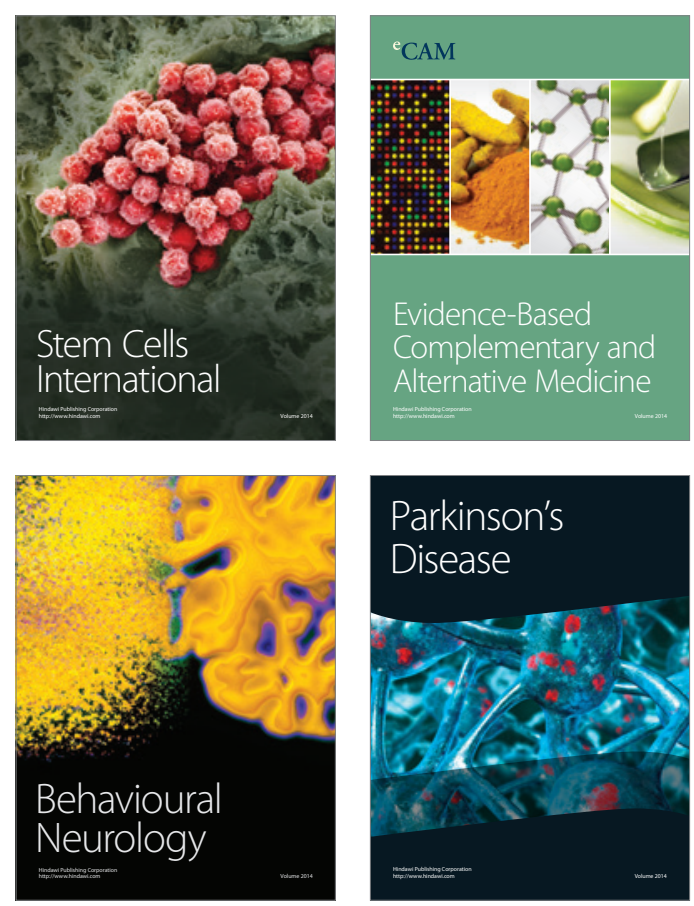

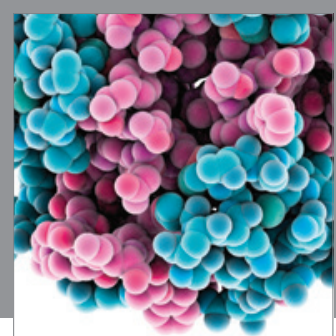

Journal of
Diabetes Research

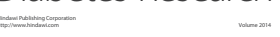

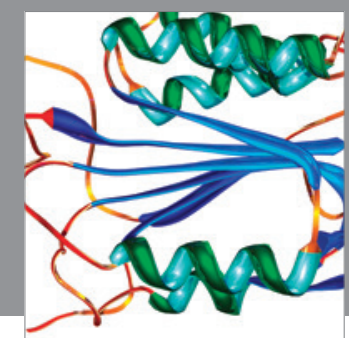

Disease Markers
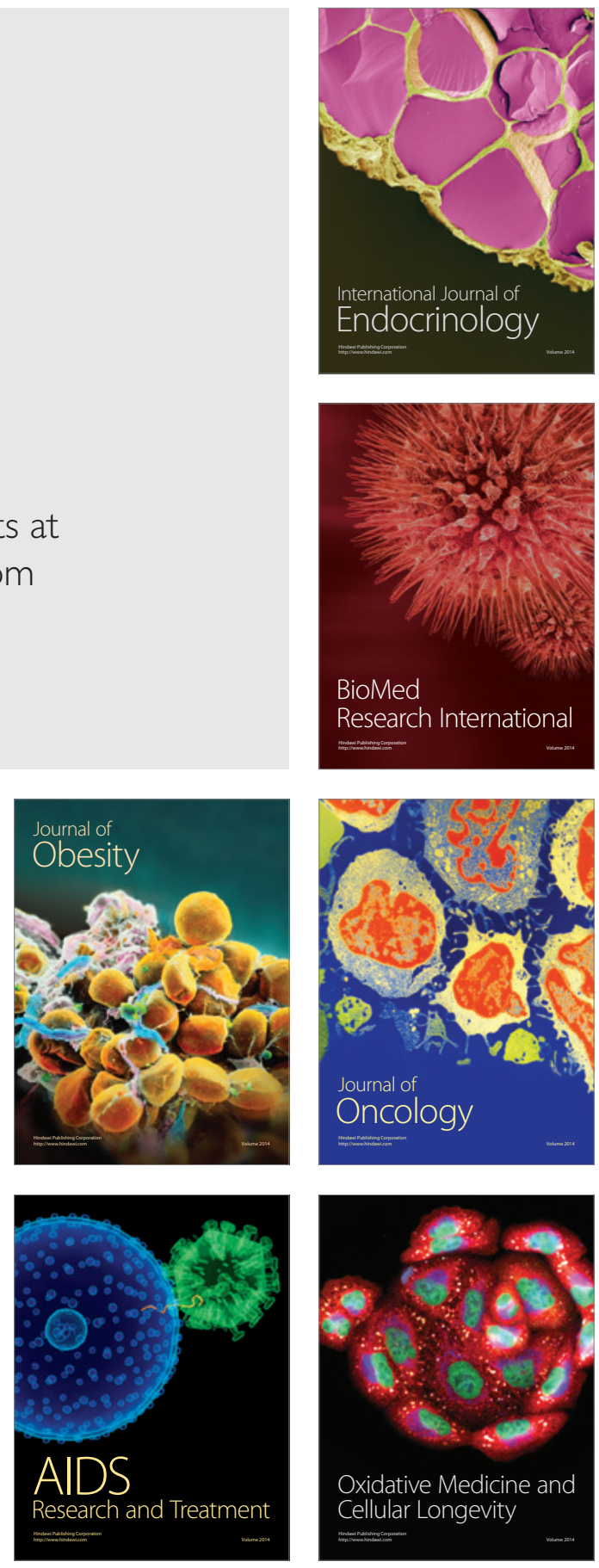